\section{Audiology Neurotology}

Baatenburg de Jong, R.J. 10

Batista, D.S. 36

Benatti, A. 21

Borkoski-Barreiro, S.A. 36

Bovo, R. 21

Calavia, D. 29

Castiglione, A. 21

de Graaff, F. 48

Falcón González, J.C. 36

Favaro, D. 21

Gabelli, C. 21

Girón, L. 29

Goedegebure, A. 10

Goverts, S.T. 48

Gräbel, S. 43
Hast, A. 16

Hocke, T. 16

Hofman, A. 10

Hoppe, U. 16

Huarte Irujo, A. 29

Huysmans, E. 48

Knopke, S. 43

Manrique-Huarte, R. 29

Manrique-Rodríguez, M. 1, 29

Martini, A. 21

Merkus, P. 48

Metselaar, M. 10

Müller, A. 16

Olze, H. 43

Padoan, E. 21

Pagliaro, M. 21
Pecorelli, S. 3

Peracino, A. 3

Pérez Plasencia, D. 36

Qazi, O.u.R. 48

Ramos de Miguel, Á. 36

Ramos-Macías, Á. 1, 36

Rigters, S.C. 10

Severi, D. 21

Smits, C. 48

Szczepek, A.J. 43

Vallesi, A. 21

Vanpoucke, F.J. 48

Velardita, C. 21

Wieringa, M.H. 10

\title{
Subject Index Vol. 21, Suppl. 1, 2016
}

Abbreviated Profile of Hearing Aid Benefit 36

Age-related hearing loss 10, 29

Aging 3, 16

Alcohol 10

Alzheimer's disease 3

Auditory outcomes 29

- rehabilitation 43

Body mass index 10

Cochlear implant 29, 36, 48

- implantation 21, 43

Cognitive decline 21

- impairment 3

Consonant-vowel-consonant recognition 48
Dementia 3

Depression 21

Determinants of hearing loss 10

Digits-in-noise 48

Direct connect 48

Discontinuous noise 48

Elderly 21, 29

- subjects 36

Glasgow Health Status Inventory 36

Health-related quality of life 36

Hearing aid 21, 29

- loss 3, 21

- use 16

'Inflamm-aging' 3
Monosyllabic score 16

Older adults 43

Personal audio cable 48

Presbycusis 10, 29

Quality of life 29, 43

Remote testing 48

Sensorial impairments 3

Smoking 10

Speech perception 16

- recognition 48

Tinnitus 43 